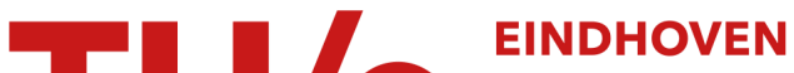 \\ UNIVERSITY OF \\ TECHNOLOGY
}

\section{Creep of chiral domain walls}

Citation for published version (APA):

Hartmann, D. M. F., Duine, R. A., Meijer, M. J., Swagten, H. J. M., \& Lavrijsen, R. (2019). Creep of chiral domain walls. Physical Review B, 100(9), [094417 ]. https://doi.org/10.1103/PhysRevB.100.094417

DOI:

10.1103/PhysRevB.100.094417

Document status and date:

Published: 11/09/2019

\section{Document Version:}

Publisher's PDF, also known as Version of Record (includes final page, issue and volume numbers)

\section{Please check the document version of this publication:}

- A submitted manuscript is the version of the article upon submission and before peer-review. There can be important differences between the submitted version and the official published version of record. People interested in the research are advised to contact the author for the final version of the publication, or visit the $\mathrm{DOI}$ to the publisher's website.

- The final author version and the galley proof are versions of the publication after peer review.

- The final published version features the final layout of the paper including the volume, issue and page numbers.

Link to publication

\section{General rights}

Copyright and moral rights for the publications made accessible in the public portal are retained by the authors and/or other copyright owners and it is a condition of accessing publications that users recognise and abide by the legal requirements associated with these rights.

- Users may download and print one copy of any publication from the public portal for the purpose of private study or research.

- You may not further distribute the material or use it for any profit-making activity or commercial gain

- You may freely distribute the URL identifying the publication in the public portal.

If the publication is distributed under the terms of Article $25 \mathrm{fa}$ of the Dutch Copyright Act, indicated by the "Taverne" license above, please follow below link for the End User Agreement:

www.tue.nl/taverne

Take down policy

If you believe that this document breaches copyright please contact us at:

openaccess@tue.nl

providing details and we will investigate your claim. 


\title{
Creep of chiral domain walls
}

\author{
Dion M. F. Hartmann $\odot,{ }^{1,}{ }^{*}$ Rembert A. Duine, ${ }^{1,2}$ Mariëlle J. Meijer, ${ }^{2}$ Henk J. M. Swagten, ${ }^{2}$ and Reinoud Lavrijsen ${ }^{2}$ \\ ${ }^{1}$ Institute for Theoretical Physics, Utrecht University, Leuvenlaan 4, NL-3584 CE Utrecht, The Netherlands \\ ${ }^{2}$ Department of Applied Physics, Eindhoven University of Technology, P.O. Box 513, 5600 MB Eindhoven, The Netherlands
}

(Received 7 January 2019; published 11 September 2019)

\begin{abstract}
Recent experimental studies of magnetic domain expansion under easy-axis drive fields in materials with a perpendicular magnetic anisotropy have shown that the domain wall velocity is asymmetric as a function of an external in-plane magnetic field. This is understood as a consequence of the inversion asymmetry of the system, yielding a finite chiral Dzyaloshinskii-Moriya interaction. Numerous attempts have been made to explain these observations using creep theory, but, in doing so, these have not included all contributions to the domain wall energy or have introduced additional free parameters. In this article we present a theory for creep motion of chiral domain walls in the creep regime that includes the most important contributions to the domain-wall energy and does not introduce new free parameters beyond the usual parameters that are included in the micromagnetic energy. Furthermore, we present experimental measurements of domain wall velocities as a function of in-plane field that are well described by our model, and from which material properties such as the strength of the Dzyaloshinskii-Moriya interaction and the demagnetization field are extracted.
\end{abstract}

DOI: 10.1103/PhysRevB.100.094417

\section{INTRODUCTION}

The interest in nanomagnetic materials has grown steadily since magnetic storage devices, such as the racetrack memory, were proposed as a new tool to meet the ever increasing demand for computer storage capacity [1-4]. For such applications the domain wall (DW) chirality is an important parameter as it affects the speed and direction of DW motion. The interfacial Dzyaloshinskii-Moriya-interaction (DMI) $[5,6]$ arises from perpendicular inversion asymmetry in the system and affects the DW chirality. Hence it is of paramount importance to be able to measure the magnitude of the DMI using a simple experimental method. The interfacial DMI is modeled as an effective field that lies in-plane (IP) and is always perpendicular to the domain wall (DW) normal, hence preferring a Néel wall [7]. Superpositioning the DMI field with an externally applied IP magnetic field could provide means of measuring it. This has lead to a boom of experimental studies on DW dynamics under the influence of an IP magnetic field [8-17].

There are several regimes of DW dynamics, determined by the strength of the DW driving force compared to the pinning force. In the flow regime the driving force is significantly higher than the pinning force and in this regime IP magnetic fields and DMI is successfully modeled by means of the Landau-Lifschitz-Gilbert equation [18-20]. In the creep regime however, the DW is considered to be mostly pinned and in local equilibrium and has a net displacement because the bias is assisted by thermal fluctuations.

The creep model was successfully implemented to interpret magnetic domain growth driven by an external magnetic field $H_{z}$ in the direction of the magnetization of one of the

*d.m.f.hartmann@uu.nl domains, resulting in the famous universal creep law for the DW velocity $v: \ln (v) \propto H_{z}^{-1 / 4}$ [21]. When introducing a magnetic field perpendicular to the magnetization direction of the domains, a modification to this creep law was proposed: $\ln (v) \propto\left(E_{\mathrm{el}} / H_{z}\right)^{1 / 4}$, where $E_{\mathrm{el}}$ is the elasticity of the DW [8]. This modification turned out to describe experimental findings well for small IP magnetic fields, but is not able to describe the high-field region [10]. Recent attempts to improve the theoretical model exposed the dispersive nature of the elasticity but compromised on universality as extra free parameters were introduced [14]. Chiral damping was proposed to explain the asymmetric component of the velocity profiles [11,15,16,22]. We contend however that in the quasistatic creep regime dynamic effects such as chiral damping should not play a significant role.

In this paper we construct a theory for motion of chiral domain walls in the creep regime which does not involve the free parameters introduced in Ref. [14]. We use it to interpret our experimental data on the DW velocity as a function of the IP magnetic field. We show that our model allows for quantitative determination of the strength of the interfacial DMI from field-driven DW creep measurements.

\section{MODEL}

In Fig. 1(a) the deformation of a DW due to a thermal fluctuation in the presence of an easy-axis driving field $H_{z}$ is illustrated. The deformation size $L$ is determined by the balance between the gained Zeeman energy from the driving field and the elastic energy cost. The deformations can be seen as nucleations whose chance of survival is determined by $L$. For such a nucleation process, Arrhenius' law tells us that the rate at which these surviving deformations will occur is determined by the height of the energy barrier $F_{\mathrm{b}}$ (i.e., the free energy at the tipping point): $\ln (v) \propto-F_{\mathrm{b}} /\left(k_{\mathrm{B}} T\right)[23,24]$. Jeudy et al. 

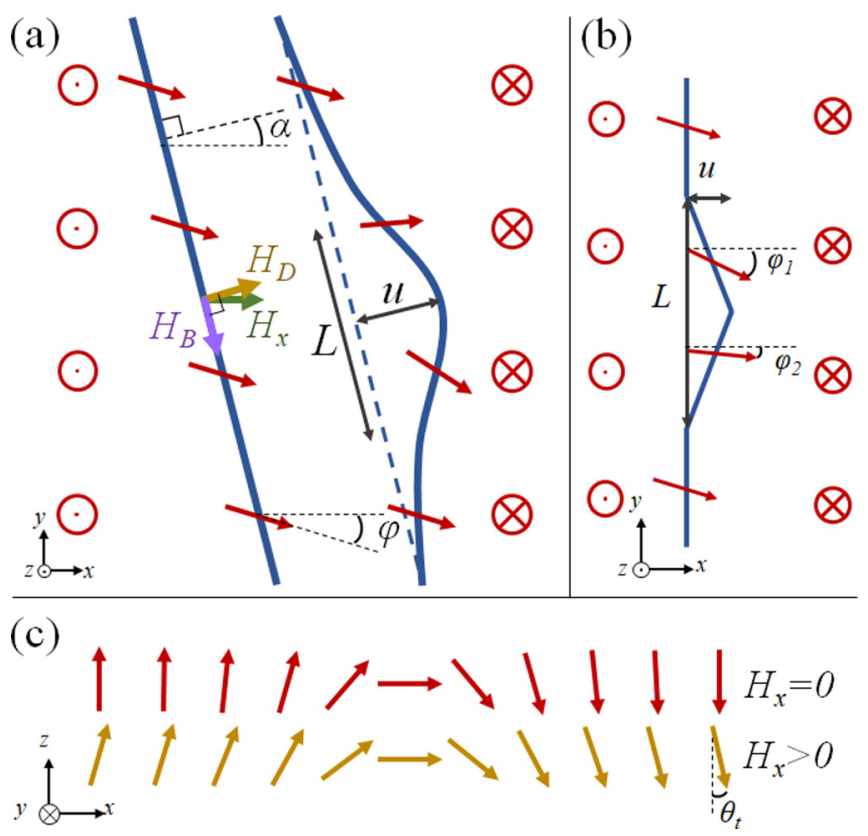

FIG. 1. (a) Top view of a DW (blue lines) that gets deformed over a length $L$ and displaced over a distance $u$ due to a thermal fluctuation. The DW can be tilted over an angle $\alpha$. The magnetization is indicated by the red vectors, which at the DW are characterized by the IP angle $\varphi$. Note that the IP magnetization changes due to the displacement, affecting the elasticity. The IP magnetic field $H_{x}$ (green) as well as the effective DMI field $H_{\mathrm{D}}$ (yellow) and effective Bloch field $H_{\mathrm{B}}$ (purple) are indicated locally. (b) Model to describe the deformation. (c) When an IP magnetic field is applied to a sample with PMA, the magnetization inside a domain tilts towards the IP magnetic field by an angle $\theta_{t}$ determined by the balance of PMA and IP magnetic field $\beta=M_{\mathrm{S}} H_{x} \cos (\varphi) / K_{\mathrm{P}}=\sin \left(\theta_{t}\right)$ (orange) compared to the $\beta=0$ case (red).

have shown that defining $F_{b}=T_{d}\left[\left(H_{d} / H_{z}\right)^{1 / 4}-1\right]$, in terms of the depinning field $H_{d}$ and an effective disorder temperature $T_{d}$, can describe the DW motion accurately in both the creep and depinning regime $[25,26]$. A recent study also used this form to capture the in-plane magnetic field effects into the depinning field [27]. Instead of postulating a form of $F_{b}$ we will determine it from micromagnetics well inside the creep regime; $F_{\mathrm{b}}=\max _{L} F(L)$. We capture the complexity of the asymmetric DW dynamics in $F(L)$. As a consequence the optimization required to determine $F_{b}$ is semianalytical. Here we introduce an insightful numerical procedure as opposed to a full analytical treatment as has been done extensively in literature $[8,14,21,25]$. This numerical approach allows us to address a plethora of effects in the underlying physics of DW dynamics in the creep regime.

$F(L)$ is composed of the elastic energy cost and the Zeeman energy gain, which depend not only on $L$, but also on the DW displacement $u$ : $F(u, L)=E_{\mathrm{el}}(u, L)+E_{\text {Zeeman }}(u, L)$. To express $u$ in terms of $L$ we use $u(L)=u_{\mathrm{c}}\left(L / L_{\mathrm{c}}\right)^{2 / 3}[21,28,29]$, where $L_{\mathrm{c}}$ is the Larkin length scale determined by minimizing the sum of the elastic and pinning energy density for $u=\xi$, and $u_{\mathrm{c}}$ is a proportionality constant. Hence the next step is to determine the elastic energy to be able to compute $L_{\mathrm{c}}$ and express $u$, and thereby $F$, in terms of $L$.
The elastic energy is defined as the difference in internal, i.e., excluding pinning and driving, energy between the domain wall before and after the deformation. Due to the application of the external IP magnetic field the DW energy density itself depends on the orientation of the DW with respect to this applied field. Furthermore, the IP magnetization of the sample at the DW is affected by the exchange interaction.

Following Blatter et al. we model the deformation as an angular shape for simplicity, see Fig. 1(b) [30]. Other shapes are possible, but this is the lowest order approximation. Note that Pellegren et al. chose an arc shape [14], but did not implement the exchange energy cost due to the kink in the connection with the straight DW segments, resulting in unphysical divergences (as demonstrated in the Supplemental Material [31]) that do not occur in our theory.

We have approximated the IP magnetization of each segment to be constant and implement a nearest neighbor exchange interaction at the bending points. The energy of the system is then minimized (numerically) over the IP magnetization angle of the two segments.

We compute the energy density of the domain wall by inserting the domain-wall solution into the micromagnetic energy functional

$$
\begin{aligned}
\mathcal{E}(\alpha, \varphi)= & 2 \sqrt{1-\beta^{2}} \frac{J}{\lambda}+M_{\mathrm{S}} \pi \lambda\left\{g(\beta) H_{\mathrm{B}} \cos ^{2}(\varphi-\alpha)\right. \\
& \left.-f(\beta)\left[H_{x} \cos (\varphi)+H_{\mathrm{D}} \cos (\varphi-\alpha)\right]\right\} .
\end{aligned}
$$

For more details see [31]. The first term is the exchange interaction $J$ over $\lambda$, the DW thickness. The second term is the demagnetization energy, expressed in terms of the effective Bloch field $H_{\mathrm{B}}$ (this energy favors a Bloch DW, hence the nomenclature), the angle $\alpha$ between the DW normal and the $x$ axis and the angle $\varphi$ the IP magnetization at the DW with the direction of the IP magnetic field, see Fig. 1(a). The third term is the Zeeman energy due to the applied IP magnetic field $H_{x}$ and the fourth is the DMI expressed in terms of an effective field $H_{\mathrm{D}}$ favoring a Néel type DW. The prefactors involving $\beta$ incorporate the tilting in the $x$ direction of the magnetic domains due to the external IP magnetic field [see Fig. 1(c)]. The functions $f$ and $g$ are given in the Supplemental Material [31].

Similarly, we obtain the Zeeman energy from the driving field $H_{z}$,

$$
E_{\text {Zeeman }}(u, L)=M_{\mathrm{S}} H_{z} t u L \sqrt{1-\beta^{2}} .
$$

Again, the factor $\sqrt{1-\beta^{2}}$ comes from the tilted domains as illustrated in Fig. 1(c). By dividing out $D\left(=M_{\mathrm{S}} \lambda H_{\mathrm{D}}\right)$ in Eqs. (1) and (2), the relevant dimensionless parameters become $\tilde{J} \equiv J \lambda^{-1} D^{-1}, \tilde{H}_{\mathrm{B}} \equiv 2 H_{\mathrm{B}} / H_{\mathrm{D}}, \tilde{H}_{x} \equiv H_{x} / H_{\mathrm{D}}$, and $\tilde{H}_{z} \equiv$ $H_{z} / H_{\mathrm{D}}$.

Using Eq. (1), we compute the optimal orientation angle of the undeformed DW $\alpha_{0}$ and the corresponding internal magnetization IP angle $\varphi_{0}$ by minimizing $\mathcal{E}(\alpha, \varphi) / \cos (\alpha)$. The factor $1 / \cos (\alpha)$ arises because we allow the DW to orient itself with respect to the IP magnetic field at the cost of elongating. For example, a mixed Bloch-Néel DW tilts its normal to better align with the external IP magnetic field. This tilting however would induce a stretching factor of $1 / \cos (\alpha)$, increasing the energy cost. This effect is illustrated in Fig. 1(a) 


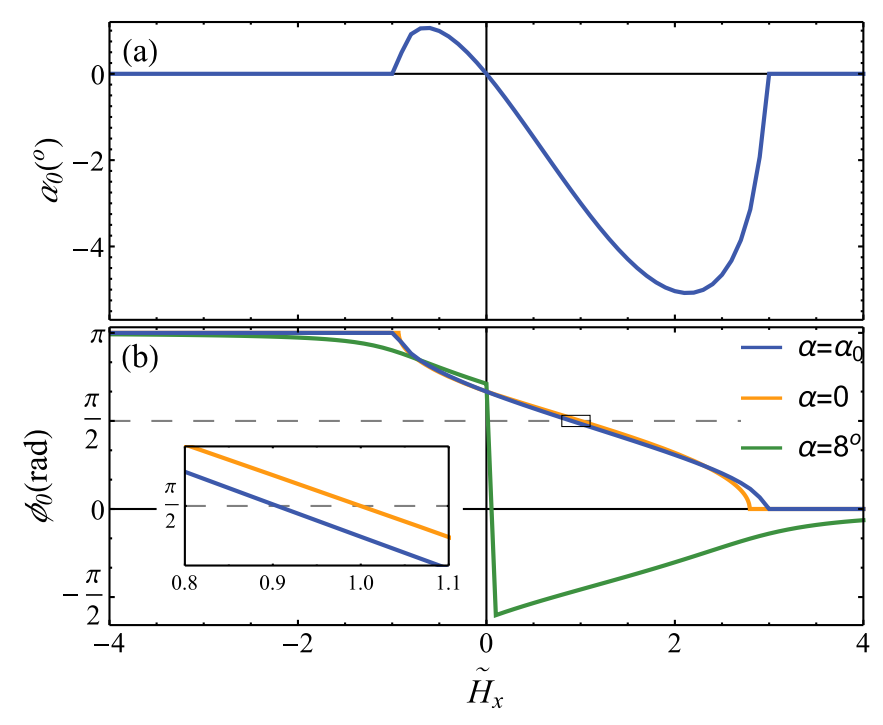

FIG. 2. $\alpha_{0}$ as a function of the applied IP magnetic field (a) and the corresponding minimized azimuthal angle of the internal magnetization $\varphi_{0}$ (b). The green curve shows the solution for $\varphi_{0}$ when $\alpha$ is fixed at $8^{\circ}$, which switches sign at $H_{x}=0$. The corresponding energy density however, remains continuous and smooth. Note that the green curve does not saturate in but converges to the Néel wall.

and the optimal angle $\alpha_{0}$ and corresponding minimized angle $\varphi_{0}$ are shown as a function of $H_{x}$ in Fig. 2. The energy of the unperturbed DW is then given by $\operatorname{Lt} \mathcal{E}\left(\alpha_{0}, \varphi_{0}\right)$.

The profile of $\varphi_{0}$ shown in Fig. 2(b) exhibits sharp kinks for both $\alpha\left(H_{x}\right)=\alpha_{0}\left(H_{x}\right)$ and $\alpha\left(H_{x}\right)=0$. This feature arises because in the energy density of Eq. (1) we neglected higher order anisotropy terms proportional to $\cos ^{n}(\varphi-\alpha)$ for $n>$ 2 which are allowed by symmetry. As a consequence, this simplified energy density yields a sharp transition in DW type from mixed Bloch-Néel to pure Néel at $f(\beta)\left(\tilde{H}_{x}-1\right)=$ $g(\beta) \tilde{H}_{\mathrm{B}}$ as demonstrated in Fig. 2 where $\varphi_{0}$ saturates to 0 or $\pi$. To effectively include for the higher order terms in the energy density, we adjust the value of $\alpha$ to some nonzero value, e.g., $\alpha=8^{\circ}$ as done by Pellegren et al. [14]. This removes the symmetry between the two deformed segments and prevents the saturation of $\varphi$. With this modification, $\varphi$ is smooth around $\varphi=0$ or $\varphi=\pi$ as demonstrated by the green curve in Fig. 2(b). We will assume $D>0$ in this paper, the results for $D<0$ are obtained by $\tilde{H}_{x} \rightarrow-\tilde{H}_{x}$.

A kink between two DW segments, as illustrated in Fig. 1(b), gives an energy cost

$$
\mathcal{E}_{\text {ben }}\left(\varphi_{1}, \varphi_{2}\right)=\frac{J \lambda}{a}\left[1-\cos \left(\varphi_{1}-\varphi_{2}\right)\right],
$$

with $\varphi_{1}$ and $\varphi_{2}$ the IP angles of the internal magnetization of the segments. Here $a$ is the distance between neighboring atoms in the magnetic layer. Due to variations in the lattice structure and to account for non-nearest neighbor interactions, an effective value of $a \sim 1 \mathrm{~nm}$ is used. The effect of $a$ on the DW dynamics is investigated in the Supplemental Material [31].
The elastic energy is computed by minimizing over $\varphi_{1}$ and $\varphi_{2}$ :

$$
\begin{aligned}
\frac{E_{\mathrm{el}}}{t}= & \min _{\varphi_{1}, \varphi_{2}}\left[\frac{L}{2} \sqrt{1+\left(\frac{2 u}{L}\right)^{2}}\left[\mathcal{E}\left(\alpha_{1}, \varphi_{1}\right)+\mathcal{E}\left(\alpha_{2}, \varphi_{2}\right)\right]\right. \\
& +\frac{J \lambda}{a}\left[3-\cos \left(\varphi_{0}-\varphi_{1}\right)-\cos \left(\varphi_{0}-\varphi_{2}\right)\right. \\
& \left.\left.-\cos \left(\varphi_{1}-\varphi_{2}\right)\right]\right]-L \mathcal{E}\left(\alpha_{0}, \varphi_{0}\right) .
\end{aligned}
$$

The first term is the length of each of the two segments of the deformed DW multiplied by their respective energy densities. $\alpha_{1}$ and $\alpha_{2}$ are the orientations of the respective segments. The second term is the bending energy for the three corners, see Fig. 1(b). The third term is the energy of the unperturbed DW.

With this expression we compute $L_{\mathrm{c}}$, express $u$ in terms of $L$, and thereby obtain $F(L)=F[u(L), L]$ from which the DW velocity is found as $\ln (v) \propto-F_{\mathrm{b}} /\left(k_{\mathrm{B}} T\right)$. For more detail, see the Supplemental Material [31] (and Refs. [32-36] therein). In summary, the derivation of the DW velocity involves multiple optimization steps to determine $\alpha_{0}, \varphi_{0}, \varphi_{1}, \varphi_{2}, L_{\mathrm{c}}$, and finally $F_{\mathrm{b}}$. Due to the complexity of the elastic energy, our results are obtained numerically.

Approximating the elasticity to be proportional to $u^{2} / L$ does allow for analytic solutions, but these are not able to fully explain recent experimental observations. For example, Je et al. approximated Eq. (4) by setting $\alpha_{0}=0, \varphi_{0}=\varphi_{1}=\varphi_{2}$ and neglecting the $\pm \arctan (2 u / L)$ in the first two terms [8]. Because Pellegren et al. have chosen a different DW profile, we cannot directly compare the expression in Eq. (1) with their results [14]. They do, however, treat $L$ as a free parameter and do not find it by optimization. Moreover, they do not account for the bending costs that we model by the terms involving $\varphi_{0}-\varphi_{1}$ and $\varphi_{0}-\varphi_{1}$ in Eq. (1).

\section{RESULTS}

In Fig. 3(a) the modeled DW velocity as a function of the applied IP magnetic field $H_{x}$ is shown for different values of $\tilde{H}_{\mathrm{B}}$ (a). Figure 3(b) shows the asymmetric component $A=\ln [v(\uparrow \downarrow) / v(\downarrow \uparrow)]$ for $\tilde{H}_{\mathrm{B}}=0.5$. The kinks in the solid lines at $\tilde{H}_{x}=1 \pm \tilde{H}_{\mathrm{B}}$ mark the saturation of internal DW magnetization angle into a Néel wall perpendicular to the IP magnetic field. These are expected from the form of Eq. (1) where we neglected terms $O\left[\cos ^{4}(\varphi)\right]$. The dashed curves are the result of setting $\alpha=8^{\circ}$ fixed to compensate for the simplified energy density.

In the high IP magnetic field regime, i.e., $\left|\tilde{H}_{x}\right|>\tilde{H}_{\mathrm{B}}$, the profile straightens out. In this regime the azimuthal angle of the internal magnetization is saturated to align with the IP magnetic field, yielding a Néel DW. Due to this saturation, the orientation dependence of the elasticity no longer varies with further increasing $\left|H_{x}\right|$. As a result, the elasticity becomes isotropic and the logarithmic increase in velocity is solely due to the gained Zeeman energy.

Note that the demonstrated asymmetry of the profile compares well with experiments [10,11,14-17,37]. Furthermore, the minimal velocity is not attained at $\tilde{H}_{x}=1$ as in the model of Je et al. [8]. 

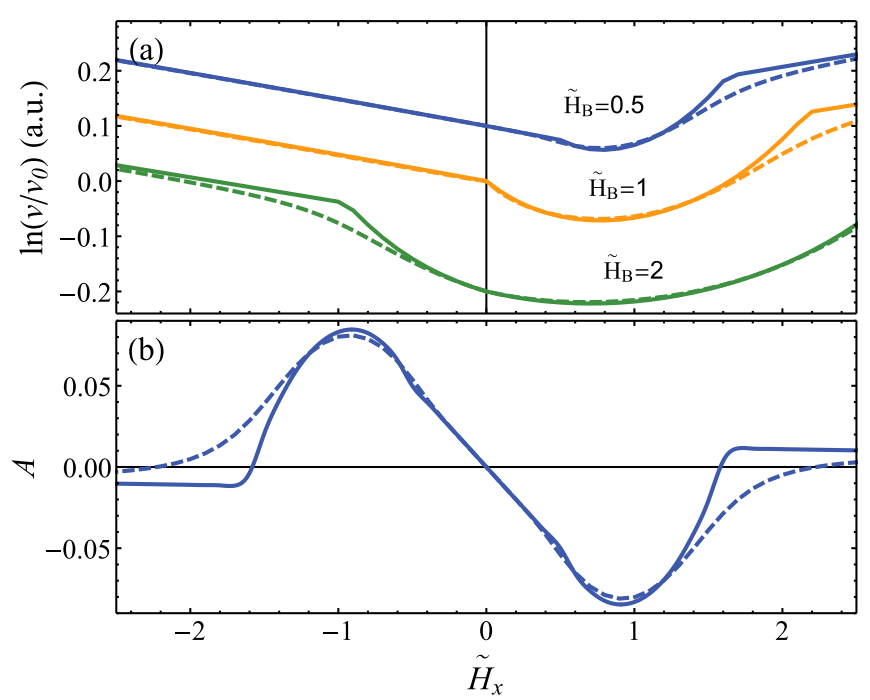

FIG. 3. Dependence of the IP magnetic field $\tilde{H}_{x}$ of the DW velocity (a) and the asymmetric component of the velocity $A$ for $\tilde{H}_{\mathrm{B}}=0.5$ (b). The profiles in (a) are given a vertical offset for clarity. The dashed lines represent the result for fixing $\alpha=8^{\circ}$. For this calculation $H_{z}=10 \mathrm{mT}$.

Note moreover that the asymmetric velocity component switches sign as $\left|\tilde{H}_{x}\right|$ increases. This feature has been observed experimentally and explained by chiral damping $[11,13,15]$. In our model there are no chiral damping effects, showing that this feature need not be an indication for chiral damping.

Finally, we compared and fitted our model to experimental data. The results are shown in Fig. 4 showing good quantitative agreement in a broad variety of samples over a wide range
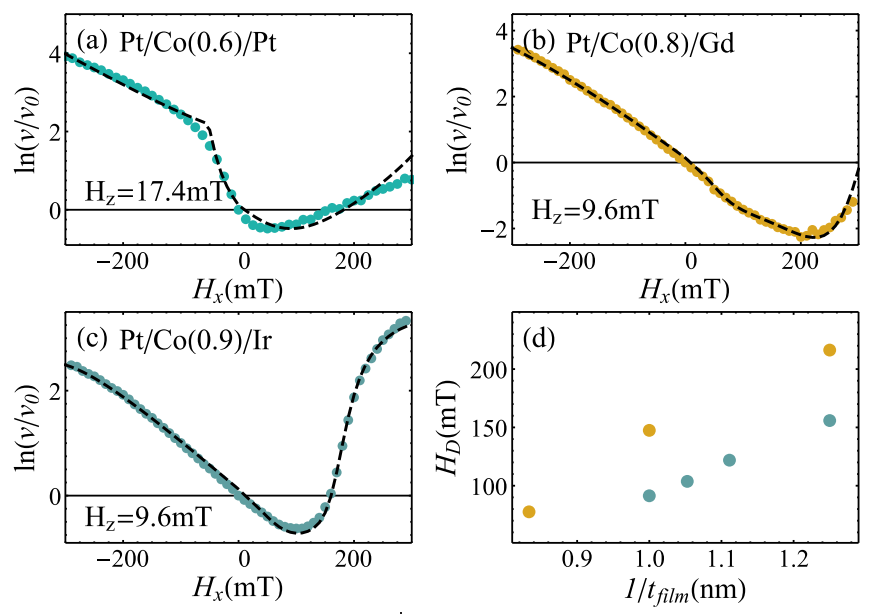

$$
\begin{array}{r|ccc}
\text { Sample } & (\mathrm{a}) & (\mathrm{b}) & (\mathrm{c}) \\
\hline H_{\mathrm{D}}(\mathrm{T}) & 0.17 & 0.22 & 0.12 \\
\tilde{H}_{\mathrm{B}} & 1.4 & 0.7 & 0.6 \\
a_{e f f}(\mathrm{~nm}) & 1.0 & 1.7 & 2.0
\end{array}
$$

FIG. 4. Fitted DW velocity curve (dashed line) to experimental data (dots) of three different samples. The data shown in (b) and (c) are obtained for this paper. The data in (a) are from Ref. [10]. The obtained fit parameters are shown in the table. (d) The $H_{\mathrm{D}}\left(1 / t_{\text {film }}\right)$ trend for a cobalt film thickness sample study in $\mathrm{Pt} / \mathrm{Co}\left(t_{\text {film }}\right) / \mathrm{Gd}$ (orange) and $\mathrm{Pt} / \mathrm{Co}\left(t_{\text {film }}\right) / \mathrm{Ir}$ (blue) stacks. The corresponding data and fits can be found in the Supplemental Material [31]. of IP magnetic fields. The asymmetric behavior is clearly demonstrated in the experiment.

We performed measurements on two different samples stacks, see Figs. 4(b) and 4(c). The samples are grown via Ar DC magnetron sputter deposition in a sputter chamber with a base pressure of $\sim 3 \times 10^{-9}$ mbar. The detailed composition of the samples is

$$
\begin{array}{l|l}
\text { Sample a } & \mathrm{SiO}_{2} / \mathrm{Ta}(4) / \mathrm{Pt}(4) / \mathrm{Co}(0.6) / \mathrm{Pt}(4) ; \\
\text { Sample b } & \mathrm{SiO}_{2} / \mathrm{Ta}(4) / \operatorname{Pt}(4) / \mathrm{Co}(0.8) / \mathrm{Gd}(3) / \operatorname{Pt}(2) ; \\
\text { Sample c } & \mathrm{SiO}_{2} / \mathrm{Ta}(4) / \operatorname{Pt}(4) / \mathrm{Co}(0.9) / \operatorname{Ir}(4) .
\end{array}
$$

The number in parentheses indicates the thickness of the layer in nanometers. These samples are representatives of the variety of the velocity profiles observed in the literature of asymmetric domain expansion experiments [8,10,12,13]. We image the magnetic domains and the expansion of those domains with a Kerr microscope setup. We use an OOP pulse magnet with a pulse length of $0.8-400 \mathrm{~ms}$ and strength up to $\pm 33 \mathrm{mT}$, and an IP magnet with a strength up to $\pm 300 \mathrm{mT}$. Furthermore, we also interpret data from previous research of Ref. [10] in Fig. 4(a). In Fig. 4(d) the obtained values for $H_{\mathrm{D}}$ are plotted as a function of the film thickness $t$ and confirm our expectation that $H_{\mathrm{D}}$ should decrease as a function of $t$ [20,31,38].

\section{CONCLUSION}

The DMI and IP magnetic field complexify DW dynamics significantly due to the orientation dependence of elasticity. To grasp and expose this complexity, we defined a model following creep theory and solving the dynamics semianalytically. The model has a profound sensitivity to DMI and demagnetization. As a result, the model provides a quantitative interpretation of experimental data of DWs that demonstrate asymmetric velocity profiles as a function of $H_{x}$.

Experimental studies that do not exhibit a kink at $H_{x}=$ $H_{\mathrm{D}} \pm 2 H_{\mathrm{B}}$ are often fitted with the constant elasticity model proposed by Je et al. [8]. In these studies the measurement range of $H_{x}$ might not be large enough to expose these kinks. Figure 4(b) demonstrates that our model resembles results from the constant elasticity model of Je et al. [8], but yields a different value of the DMI: at $H_{x}=H_{D}$, the velocity is not minimized.

The parameter $\alpha$ has been set to a fixed value to account for the omission of higher order anisotropy terms in the energy density. As a result the angle $\varphi$ will not saturate for large $H_{x}$. Previous research used $\alpha$ as a fitting parameter to account for roughness [14]. If roughness forces the DW to tilt, the tilting angle is not fixed to one value. Hence a fixed value of $\alpha$ should not be interpreted as a physical tilting of the DW.

We remark that assuming $\varphi$ to be constant along an axis normal to the DW is only a first approximation. For a mixed Bloch-Néel DW, $\varphi$ will adjust so that the magnetization aligns with the IP magnetic field well inside the domains, but does not at the DW. As $\varphi$ plays a key role in the DW dynamics, future research could focus on the exact behavior of $\varphi$.

In recent publications the asymmetric shape of the DW velocity profile as a function of $H_{x}$ is used as an argument for significant effect of chiral damping on the DW dynamics $[11,15,16]$. However, our model demonstrates a similar 
asymmetry without chiral damping. Furthermore, in the quasistatic creep regime dynamic effects such as chiral damping should not affect creep motion.

The comparison experimental data demonstrates the broad applicability of our model. Future research could apply our model to an extensive sample study to investigate the effects of sample growth parameters and layer thickness on parameters of the model such as the effective lattice spacing $a$. Furthermore, measurements over a broader range in $H_{z}$ could be performed to test the universality.

\section{ACKNOWLEDGMENTS}

R.A.D. is member of the D-ITP consortium, a program of the Dutch Organisation for Scientific Research (NWO) that is funded by the Dutch Ministry of Education, Culture and Science (OCW). This work is funded by the European Research Council (ERC). This work is part of the research programme of the Foundation for Fundamental Research on Matter (FOM), which is part of the Dutch Organisation for Scientific Research (NWO).
[1] S. S. Parkin, M. Hayashi, and L. Thomas, Science 320, 190 (2008).

[2] K.-W. Moon, D.-H. Kim, S.-C. Yoo, S.-G. Je, B. S. Chun, W. Kim, B.-C. Min, C. Hwang, and S.-B. Choe, Sci. Rep. 5, 9166 (2015).

[3] D. A. Allwood, G. Xiong, C. Faulkner, D. Atkinson, D. Petit, and R. Cowburn, Science 309, 1688 (2005).

[4] M. Hayashi, L. Thomas, R. Moriya, C. Rettner, and S. S. Parkin, Science 320, 209 (2008).

[5] I. Dzyaloshinsky, J. Phys. Chem. Solids 4, 241 (1958).

[6] T. Moriya, Phys. Rev. 120, 91 (1960).

[7] A. Fert and P. M. Levy, Phys. Rev. Lett. 44, 1538 (1980).

[8] S.-G. Je, D.-H. Kim, S.-C. Yoo, B.-C. Min, K.-J. Lee, and S.-B. Choe, Phys. Rev. B 88, 214401 (2013).

[9] O. Boulle, S. Rohart, L. D. Buda-Prejbeanu, E. Jué, I. M. Miron, S. Pizzini, J. Vogel, G. Gaudin, and A. Thiaville, Phys. Rev. Lett. 111, 217203 (2013).

[10] R. Lavrijsen, D. M. F. Hartmann, A. van den Brink, Y. Yin, B. Barcones, R. A. Duine, M. A. Verheijen, H. J. M. Swagten, and B. Koopmans, Phys. Rev. B 91, 104414 (2015).

[11] E. Jué, C. Safeer, M. Drouard, A. Lopez, P. Balint, L. BudaPrejbeanu, O. Boulle, S. Auffret, A. Schuhl, A. Manchon et al., Nat. Mater. 15, 272 (2016).

[12] M. Vaňatka, J.-C. Rojas-Sánchez, J. Vogel, M. Bonfim, M. Belmeguenai, Y. Roussigné, A. Stashkevich, A. Thiaville, and S. Pizzini, J. Phys.: Condens. Matter 27, 326002 (2015).

[13] D. Lau, V. Sundar, J.-G. Zhu, and V. Sokalski, Phys. Rev. B 94, 060401(R) (2016).

[14] J. P. Pellegren, D. Lau, and V. Sokalski, Phys. Rev. Lett. 119, 027203 (2017).

[15] D.-Y. Kim, M.-H. Park, Y.-K. Park, J.-S. Kim, Y.-S. Nam, D.-H. Kim, S.-G. Je, B.-C. Min, and S.-B. Choe, NPG Asia Mater. 10, e464 (2018).

[16] D. Lau, J. P. Pellegren, H. Nembach, J. Shaw, and V. Sokalski, Phys. Rev. B 98, 184410 (2018).

[17] D.-Y. Kim, M.-H. Park, Y.-K. Park, J.-S. Kim, Y.-S. Nam, H.S. Hwang, D.-H. Kim, S.-G. Je, B.-C. Min, and S.-B. Choe, Phys. Rev. B 97, 134407 (2018).

[18] L. Landau and E. Lifshitz, On the theory of the dispersion of magnetic permeability in ferromagnetic bodies, Perspectives in Theoretical Physics (Elsevier, 1992).
[19] Z. Li and S. Zhang, Phys. Rev. Lett. 92, 207203 (2004).

[20] A. Thiaville, S. Rohart, Émilie Jué, V. Cros, and A. Fert, Europhys. Lett. 100, 57002 (2012).

[21] S. Lemerle, J. Ferré, C. Chappert, V. Mathet, T. Giamarchi, and P. Le Doussal, Phys. Rev. Lett. 80, 849 (1998).

[22] K.-W. Kim and H.-W. Lee, Nat. Mater. 15, 253 (2016).

[23] S. Arrhenius, Z. Phys. Chem. 4U, 226 (1889).

[24] S. Arrhenius, Z. Phys. Chem. 4U, 96 (1889).

[25] V. Jeudy, A. Mougin, S. Bustingorry, W. Savero Torres, J. Gorchon, A. B. Kolton, A. Lemaître, and J.-P. Jamet, Phys. Rev. Lett. 117, 057201 (2016).

[26] V. Jeudy, R. Diaz Pardo, W. Savero Torres, S. Bustingorry, and A. B. Kolton, Phys. Rev. B 98, 054406 (2018).

[27] K. Shahbazi, J.-V. Kim, H. T. Nembach, J. M. Shaw, A. Bischof, M. D. Rossell, V. Jeudy, T. A. Moore, and C. H. Marrows, Phys. Rev. B 99, 094409 (2019).

[28] M. Kardar and D. R. Nelson, Phys. Rev. Lett. 55, 1157 (1985).

[29] D. S. Fisher and D. A. Huse, Phys. Rev. B 43, 10728 (1991).

[30] G. Blatter, M. V. Feigel'man, V. B. Geshkenbein, A. I. Larkin, and V. M. Vinokur, Rev. Mod. Phys. 66, 1125 (1994).

[31] See Supplemental Material at http://link.aps.org/supplemental/ 10.1103/PhysRevB.100.094417 for detailed derivations, used standard parameter values and an elaboration on creep theory and the experimental method.

[32] S. Tarasenko, A. Stankiewicz, V. Tarasenko, and J. Ferré, J. Magn. Magn. Mater. 189, 19 (1998).

[33] A. Malozemoff and J. Slonczewski, Magnetic Domain Walls in Bubble Materials (Academic Press, New York, 1979), p. 382.

[34] A. Hubert, Theorie der Domänenwände in Geordneten Medien (Springer, Berlin, 1974).

[35] We are very grateful to Maxim Faber for pointing this out.

[36] M. Belmeguenai, Y. Roussigné, H. Bouloussa, S. M. Chérif, A. Stashkevich, M. Nasui, M. S. Gabor, A. Mora-Hernández, B. Nicholson, O.-O. Inyang et al., Phys. Rev. Appl. 9, 044044 (2018).

[37] A. Hrabec, N. A. Porter, A. Wells, M. J. Benitez, G. Burnell, S. McVitie, D. McGrouther, T. A. Moore, and C. H. Marrows, Phys. Rev. B 90, 020402(R) (2014).

[38] P. J. Metaxas, J. P. Jamet, A. Mougin, M. Cormier, J. Ferré, V. Baltz, B. Rodmacq, B. Dieny, and R. L. Stamps, Phys. Rev. Lett. 99, 217208 (2007). 